\title{
ALMOST COMPLEX STRUCTURES ON TENSOR BUNDLES
}

\author{
A. J. LEDGER \& K. YANO
}

\section{Introduction}

It is well known that the tangent bundle of a $C^{\infty}$ manifold $M$ admits an almost complex structure if $M$ admits an affine connection [1], [5] or an almost complex structure [7], [8]. The main purpose of this paper is to investigate a similar problem for tensor bundles $T_{s}^{r} M$. We prove that if a Riemannian manifold $M$ admits an almost complex structure then so does $T_{s}^{r} M$ provided $r+s$ is odd. If $r+s$ is even a further condition is required on $M$. The proofs depend on some generalizations of the notions of lifting vector fields and derivations on $M$, which were defined previously only for tangent bundles and cotangent bundles [4], [7], [8], [9], [10].

\section{Notations and definitions}

(i) $M$ is a $C^{\infty}$ paracompact manifold of finite dimension $n$.

(ii) $F(M)$ is the ring of real-valued $C^{\infty}$ functions on $M$.

(iii) For $r+s>0, T_{s}^{r} M$ is the bundle over $M$ of tensors of type $(r, s)$, contravariant of order $r$ and covariant of order $s . \pi$ is the projection of $T_{s}^{r} M$ onto $M$. We write $T_{0}^{r} M=T^{r} M, T_{s}^{0} M=T_{s} M$.

(iv) $\mathscr{T}_{s}^{r}(M)$ is the module over $F(M)$ of $C^{\infty}$ tensor fields of type $(r, s)$. We write $\mathscr{T}_{0}^{r}(M)=\mathscr{T}^{r}(M), \mathscr{T}_{s}^{0}(M)=\mathscr{T}_{s}(M)$, and $\mathscr{T}_{0}^{0}(M)=F(M)$. $\mathscr{T}(M)$ is the direct sum $\sum_{r, s} \mathscr{T}_{s}^{r}(M) . T_{p}$ is the value at $p \in M$ of a tensor field $T$ on $M$, and $\stackrel{r, s}{\mathscr{T}}_{s}^{r}(p)$ is the vector space of tensors of type $(r, s)$ at $p$.

(v) Let $S \in \mathscr{T}_{r}^{s}(p)$ and $T \in \mathscr{T}_{s}^{r}(p)$. Then the real number $S(T)=T(S)$ is defined, in the usual way, by contraction. It follows that if $S \in \mathscr{T}_{r}^{s}(M)$ then $S$ is a differentiable function on $T_{s}^{r} M$.

(vi) A map $D: \mathscr{T}(M) \rightarrow \mathscr{T}(M)$ is a derivation on $M$ if

(a) $D$ is linear with respect to constant coefficients,

(b) for all $r, s, D \mathscr{T}_{s}^{r}(M) \subset \mathscr{T}_{s}^{r}(M)$,

(c) for all $C^{\infty}$ tensor fields $T_{1}$ and $T_{2}$ on $M$,

$$
D\left(T_{1} \otimes T_{2}\right)=\left(D T_{1}\right) \otimes T_{2}+T_{1} \otimes D T_{2},
$$

Communicated August 15, 1967. 
(d) $D$ commutes with contraction.

A derivation is determined by its action on $F(M)$ and $\mathscr{T}^{1}(M)$. In particular, $\mathscr{T}_{1}^{1}(M)$ may be identified with the set of derivations which map $F(M)$ to zero. The set of derivations on $M$ forms a module $\mathscr{D} M$ over $F(M)$.

(vii) The notation for covariant derivatives and curvature tensors is that of [2]. The linear connections considered on $M$ are assumed to have zero torsion.

\section{Vector fields on $T_{s}^{r} M$}

In this section we show how vector fields on $T_{s}^{r} M$ can be induced from vector fields, tensor fields of type $(r, s)$, and derivations on $M$.

We first prove a lemma which, together with its corollary, will be of use later.

Lemma 1. Let $p \in M$ and $S \in \pi^{-1}(p)$. If $W$ is a vertical vector at $S$ (i.e. tangential to $\pi^{-1}(p)$ at $S$ ) and $W(\alpha)=0$ for all $\alpha \in \mathscr{T}_{r}^{s}(p)$ then $W=0$.

Proof. The vector space $\mathscr{T}_{r}^{s}(p)$ is dual to $\mathscr{T}_{s}^{r}(p)$ and hence $\alpha$ contains a system of coordinates on $\pi^{-1}(p)$. The result follows immediately.

Corollary 1. Let $W \in \mathscr{T}^{1}\left(T_{s}^{r} M\right)$. If $W(\alpha)=0$ for all $\alpha \in \mathscr{T}_{r}^{s}(M)$ then $W=0$.

Proof. The assumption on $W$ implies that for $\beta \in \mathscr{T}_{r-1}^{s}(M)$ and $f \in F(M)$,

$$
0=\frac{1}{2} W\left(d f^{2} \otimes \beta\right)=W((f \circ \pi) d f \otimes \beta)=W(f \circ \pi) d f \otimes \beta .
$$

Hence $d \pi W=0$, and so $W$ is a vertical vector field. Thus $W=0$ by Lemma 1 , the values of $W$ on the zero section of $\mathscr{T}_{s}^{r} M$ being zero by continuity.

Proposition 1. Let $T \in \mathscr{T}_{s}^{r}(M)$. Then there is a unique $C^{\infty}$ vector field $T^{v}$ on $T_{s}^{r} M$ such that for $\alpha \in \mathscr{T}_{r}^{s}(M)$,

$$
T^{v}(\alpha)=\alpha(T) \circ \pi
$$

Proof. For $p \in M, \pi^{-1}(p)$ is a vector space and so $T_{p}$ determines a unique vertical vector field $T_{p}^{v}$ on $\pi^{-1}(p)$ such that for $\alpha \in \mathscr{T}_{r}^{s}(p), T_{p}^{v}(\alpha)=\alpha\left(T_{p}\right)$. The cross section $T$ on $T_{s}^{r} M$ then determines a $C^{\infty}$ vertical vector field which satisfies (1). $T^{v}$ will be called the vertical lift of $T$.

Corollary 2. Let $S \in \pi^{-1}(p)$, and let $T_{S}^{v}$ be the value of $T^{v}$ at $S$. Then the map $T_{p} \rightarrow T_{S}^{v}$ is a linear isomorphism of $\pi^{-1}(p) \rightarrow\left(\pi^{-1}(p)\right)_{S}$, where $\left(\pi^{-1}(p)\right)_{S}$ is the tangent space to the fibre $\pi^{-1}(p)$ at $S$.

Proposition 2. Let $D$ be a derivation on $M$. Then there is a unique vector field $\bar{D}$ on $T_{s}^{r} M$ such that for $\alpha \in \mathscr{T}_{r}^{s}(M)$

$$
\bar{D} \alpha=D \alpha \text {. }
$$


Proof. Let $\left\{x^{i}\right\}(i=1,2, \cdots, n)$ be a coordinate system on a neighbourhood $U$ of $p \in M$, and $\left\{\omega^{\theta}\right\}\left(\theta=1,2, \cdots, n^{r+s}\right)$ a basis for $\mathscr{T}_{i}^{s}(U)$. Then $\left\{x^{i} \circ \pi, \omega^{\theta}\right\}$ is a coordinate system on $\pi^{-1}(U)$. Define $\bar{D}$ on $\pi^{-1}(U)$ by

$$
\begin{aligned}
\bar{D}\left(x^{i} \circ \pi\right) & =\left(D x^{i}\right) \circ \pi, \\
\bar{D}\left(\omega^{\theta}\right) & =D\left(\omega^{\theta}\right) .
\end{aligned}
$$

Thus a $C^{\infty}$ vector field $\bar{D}$ is defined on $\pi^{-1}(U)$. Moreover, for $\alpha \in \mathscr{T}_{r}^{s}(U)$ we have $\bar{D} \alpha=D \alpha$. Hence, using Corollary 1 , it follows that $\bar{D}$ is defined over $T_{s}^{r} M$ as the unique solution of (2).

Corollary 3. If $f \in F(M)$ then $\bar{D}(f \circ \pi)=(D f) \circ \pi$.

Corollary 4. $\bar{D}$ is a vertical vector field if and only if $D \in \mathscr{T}_{1}^{1}(M)$.

Corollary 5. If $D_{1}, D_{2}$ are derivations on $M$, and $f_{1}, f_{2} \in F(M)$, then $f_{1} D_{1}+f_{2} D_{2}$ is a derivation on $M$, and

$$
\overline{f_{1} D_{1}+f_{2} D_{2}}=\left(f_{1} \circ \pi\right) \bar{D}_{1}+\left(f_{2} \circ \pi\right) \bar{D}_{2} .
$$

Thus if $F(M)$ is identified with $F(M) \circ \pi=\{f \circ \pi: f \in F(M)\}$ then $D \rightarrow \bar{D}$ is a linear map of $\mathscr{D} M \rightarrow \mathscr{T}^{\mathrm{l}}\left(T_{s}^{r} M\right)$.

Corollary 6. If $p \in M$ and $A \in \mathscr{T}_{1}^{1}(p)$ then for $S \in T_{s}^{\gamma}(p)$,

$$
\bar{A}_{S}=-(A S)_{S}^{v}
$$

where the suffix $S$ denotes evaluation at $S$.

Proof. Let $\alpha \in \mathscr{T}_{r}^{s}(p)$. Then

$$
\bar{A}_{S}(\alpha)=(A \alpha)(S)=-(A S)_{S}^{v}(\alpha) .
$$

The result follows from Lemma 1.

Corollary 7. Let $X \in \mathscr{T}^{1}(M)$ and $\mathscr{L}_{X}$ denote Lie derivation with respect to $X$. Then $\overline{\mathscr{L}}_{X}$ is a vector field on $T_{s}^{r} M$. In conformity with the notation of [4], [8], [9], [10], we call $\overline{\mathscr{L}}_{X}$ the complete lift of $X$ and write $\overline{\mathscr{L}}_{X}=X^{c}$.

Remark 1. If $f \in F(M)$ then

$$
\mathscr{L}_{f X}=f \mathscr{L}_{X}-X \otimes d f,
$$

where $X \otimes d f$ is regarded as a derivation on $M$. Thus

$$
(f X)^{c}=(f \circ \pi) X^{c}-\overline{X \otimes d f} .
$$

Now if $T_{s}^{r} M$ is the tangent bundle $T^{1} M$ then for $\alpha \in \mathscr{T}_{1}(M)$,

$$
\overline{X \otimes d f}(\alpha)=-\alpha(X) d f .
$$

Hence by Proposition 1, 


$$
\overline{X \otimes d f}=-d f X^{v}
$$

where $X^{v}$ is the vertical lift of $X$ to $T^{1} M$. We then have

$$
(f X)^{c}=f X^{c}+d f X^{v} .
$$

Equation (7) was used extensively in [8] but does not appear to extend to tensor bundles of high order. Equation (6) is perhaps a useful generalization.

Lemma 2. Let $p \in M$ and $A \in \mathscr{T}_{1}^{1}(p)$. Suppose there exist non-negative integers $a$ and $b$, not both zero, such that $A \mathscr{T}_{b}^{a}(p)=0$. Then $A=k I$ where $k$ is some real number. If $a \neq b$ then $A=0$.

Proof. We prove the lemma for the case $a>0$. The proof for $a=0$ and $b>0$ is essentially the same with covariance and contravariance exchanged.

Let $S \in \mathscr{T}_{b}^{a-1}(p)$ be non-zero, and let $X \in \mathscr{T}^{1}(p)$. Then

$$
A S \otimes X+S \otimes A X=0 .
$$

Choose $\omega \in \mathscr{T}_{a-1}^{b}(p)$ such that $\omega(S) \neq 0$. Then $(A-k I) X=0$, where $k=-\omega(A S) / \omega(S)$. It follows immediately that $A=k I$. Then for $T \in \mathscr{T}_{b}^{a}(p)$

$$
0=A T=k(a-b) T .
$$

Hence, if $a \neq b$ then $k=0$ and $A=0$.

Remark 2. $A=k I$ for some $k$ is a necessary and sufficient condition for $A \mathscr{T}_{a}^{a}(p)=0, a \neq 0$.

Corollary 8. Let $D \in \mathscr{D M}$ and suppose there exist non-negative integers a and $b$, not both zero, such that $D \mathscr{T}_{b}^{a}(M)=0$. Then $D=f I$, where $f \in F(M)$. If $a \neq b$ then $D=0$.

Proof. Let $h \in F(M)$ and $T \in \mathscr{T}_{b}^{a}(M)$. Then

$$
(D h) T=0 \text {. }
$$

It follows immediately that $D F(M)=0$ and hence $D \in \mathscr{T}_{1}^{1}(M)$. Then by Lemma 2, $D=f I$ for some $f \in F(M)$, and if $a \neq b$, then $f$ is zero by Lemma 2. This completes the proof.

Remark 3. $D=f I$ for some $f \in F(M)$ is a necessary and sufficient condition for $D \mathscr{T}_{a}^{a}(M)=0, a \neq 0$.

Corollary 9. The map $D \rightarrow \bar{D}$ of $\mathscr{D} M \rightarrow \mathscr{T}^{1}\left(T_{s}^{r} M\right)$ is a monomorphism when $r \neq s$ and has kernel $\{f I: f \in F(M)\}$ when $r=s$.

Proof. This follows from Corollaries 1, 5 and 8.

Corollary 10. If $r \neq s$ then $T_{s}^{r} M$ admits a vertical vector field which vanishes only on the zero section of $T_{s}^{r} M$.

Proof. The vector field $\bar{I}$ has the required properties.

Corollary 11. Let $p \in M, A \in \mathscr{T}_{1}^{1}(p)$ and $T \in \mathscr{T}_{s}^{r}(P), r \neq s$. Then $\bar{A}=T^{v}$ implies $A=0$ and $T=0$. 
Proof. Suppose $\bar{A}=T^{v}$. Then by Corollaries 2 and $6, A S=-T$ for all $S \in \mathscr{T}_{s}^{r}(p)$. Since $A$ is linear it follows that $T=0$ and $A \mathscr{T}_{s}^{r}(p)=0$. Hence $A=0$ by Lemma 2 .

Suppose now that $\nabla$ is a linear connection (with zero torsion) on $M$, and let $X \in \mathscr{T}^{1}(M)$. Then $\nabla X \in \mathscr{T}_{1}^{1}(M)$, and hence, by Corollary $4, \overline{\nabla X}$ is a $C^{\infty}$ vertical vector field on $T_{s}^{r} M$.

Another $C^{\infty}$ vector field $\bar{\nabla}_{X}$ on $T_{s}^{r} M$ is determined by the derivation $\nabla_{X}$. In conformity with [4] we write $\bar{\nabla}_{X}=X^{h}$, and call $X^{h}$ the horizontal lift of $X$. If $f \in F(M)$ then using Corollary 3 ,

$$
X^{n}(f \circ \pi)=\bar{\nabla}_{X}(f \circ \pi)=\left(\nabla_{X} f\right) \circ \pi=(X f) \circ \pi .
$$

Hence

$$
d \pi X^{h}=X
$$

The horizontal lift clearly satisfies

$$
(f X+g Y)^{h}=(f \circ \pi) X^{h}+(g \circ \pi) Y^{h},
$$

for $f, g \in F(M)$ and $X, Y \in \mathscr{T}^{1}(M)$. Thus the horizontal lift is a linear map of $\mathscr{T}^{1}(M) \rightarrow \mathscr{T}^{1}\left(T_{s}^{r} M\right)$ if, as before, $F(M)$ and $F(M) \circ \pi$ are identified. Since $\bar{\nabla}_{X}=0$ if and only if $X=0$, the horizontal lift is a monomorphism, and so determines a horizontal subspace $H_{S}$ of dimension $n(=\operatorname{dim} M)$ at each point $S \in T_{s}^{r} M$. Then $C^{\infty}$ distribution $H$ on $T_{s}^{r} M$ so obtained is usually called the horizontal distribution determined by the connection $\nabla$.

If $S \in T_{s}^{r} M$ then the tangent space $\left(T_{s}^{r} M\right)_{S}$ is the direct sum $V_{S}+H_{S}$, where $V_{S}$ is the subspace of vertical vectors at $S$. Thus, if $W \in\left(T_{s}^{r} M\right)_{S}$ then

$$
W=h(W)+v(W)
$$

where $h$ and $v$ are the projections onto the horizontal and vertical subspaces at $S$. Clearly $X^{h}=h\left(X^{h}\right)$ and $T^{v}=v\left(T^{v}\right)$ for any vector $X$ and tensor $T$ of type $(r, s)$ at $\pi(S)$.

\section{Lie brackets}

We now determine, for later use, the Lie brackets of some particular types of vector fields on $T_{s}^{r} M$. These results generalize some of those already obtained for tangent bundles and cotangent bundles [1], [4], [7], [8], [9], [10].

Lemma 3. Let $T_{1}, T_{2} \in \mathscr{T}_{s}^{r}(M)$ and $X, X_{1}, X_{2} \in \mathscr{T}^{1}(M)$, and let $D, D_{1}, D_{2}$, $A$ be derivations on $M$, where $A \in \mathscr{T}_{1}^{1}(M)$. Let $R$ denote the curvature tensor field of the connection $\nabla$. Then

$$
\left[T_{1}^{v}, T_{2}^{v}\right]=0
$$




$$
\begin{aligned}
{\left[\bar{D}_{1}, \bar{D}_{2}\right] } & =\left[\overline{D_{1}, D_{2}}\right], \\
{\left[\bar{D}, T^{v}\right] } & =(D T)^{v}, \\
{\left[X^{h}, T^{v}\right] } & =\left(\nabla_{X} T\right)^{v}, \\
{\left[X_{1}^{h}, X_{2}^{h}\right] } & \left.=\overline{R\left(X_{1}, X_{2}\right.}\right)+\left[X_{1}, X_{2}\right]^{h}, \\
{\left[X^{h}, \bar{A}\right] } & =\overline{\nabla_{X} A}, \\
{\left[X_{1}^{c}, X_{2}^{c}\right] } & =\left[X_{1}, X_{2}\right]^{c} .
\end{aligned}
$$

Proof. Several equations can be proved by application of Corollary 1.

If $p \in M$ then $\pi^{-1}(p)$ is a vector space, and has the structure of an abelian Lie group. If $S \in \mathscr{T}_{s}^{r}(M)$ then $S^{v}$ is an invariant vector field on $\pi^{-1}(p)$ and equation (9) follows immediately.

We have, from Proposition 2,

$$
\left[\bar{D}_{1}, \bar{D}_{2}\right] \alpha=\left(\bar{D}_{1} \bar{D}_{2}-\bar{D}_{2} \bar{D}_{1}\right) \alpha=\left[D_{1}, D_{2}\right] \alpha .
$$

Since $\left[D_{1}, D_{2}\right]$ is a derivation on $M$, from Proposition 2 we have

$$
\left[D_{1}, D_{2}\right] \alpha=\left[\overline{D_{1}, D_{2}}\right] \alpha,
$$

and hence equation (10).

$$
\left[\bar{D}, T^{v}\right] \alpha=(D(\alpha(T))-(D \alpha)(T)) \circ \pi=(\alpha(D T)) \circ \pi=(D T)^{v}(\alpha),
$$

which gives equation (11). Since $X^{n}=\bar{\nabla}_{X}$, equation (12) is a special case of (11).

Since $R\left(X_{1}, X_{2}\right) \in \mathscr{T}_{1}^{1}(M)$, we have

$$
\left[X_{1}^{h}, X_{2}^{h}\right]=\left[\bar{\nabla}_{X_{1}}, \bar{\nabla}_{X_{2}}\right]=\left[\overline{\bar{V}_{X_{1}}, \nabla_{X_{2}}}\right]=\overline{R\left(X_{1}, X_{2}\right)}+\bar{\nabla}_{\left[X_{1}, X_{2}\right]},
$$

from which follows immediately equation (13).

$$
\left[X^{h}, \bar{A}\right] \alpha=\nabla_{X}(A \alpha)-A\left(\nabla_{X} \alpha\right)=\left(\nabla_{X} A\right) \alpha=\left(\overline{\nabla_{X} A}\right) \alpha,
$$

which gives equation (14). Since $X^{c}=\overline{\mathscr{L}}_{X}$, equation (15) is a special case of (10).

\section{Almost complex structures}

We now consider the main problem, that is, to determine a class of tensor bundles which admit almost complex structures. For this purpose it is sufficient to consider contravariant tensor bundles since a Riemannian metric tensor field induces a fibre preserving diffeomorphism of $T_{s}^{r} M \rightarrow T^{r+s} M$. Also 
the tangent bundle $T^{1} M$ of a Riemannian space always admits an almost complex structure [1], [5]. Hence we shall restrict attention to $T^{r} M, r>1$.

Lemma 4. Let $\nabla$ and $g$ be, respectively, a symmetric connection and a Riemannian metric tensor field on $M$, and $E \in \mathscr{T}^{r-1}(M)$ be nowhere zero on $M$. Then $T^{r} M$ admits three distributions which are mutually orthogonal with respect to a Riemannian metric tensor field $\bar{g}$ induced on $T^{r} M$ by $\nabla$ and $g$.

Proof. For each $p \in M$ a scalar product $\langle$, $\rangle$ is defined on the vector space $\pi^{-1}(p)$ by $\left\langle T_{1}, T_{2}\right\rangle=t_{1}\left(T_{2}\right)$, where, for any tensor $T$ with components

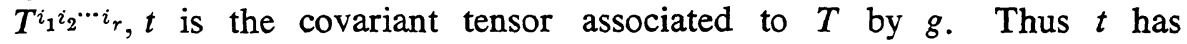
components

$$
t_{i_{1} i_{2} \cdots i_{r}}=T^{j_{1} j_{2} \cdots j_{r}} g_{i_{1} j_{1}} g_{i_{2} j_{2}} \cdots g_{i_{r} j_{r}},
$$

where each repeated suffix indicates summation over its range. If $S \in T^{r} M$, then a scalar product, denoted by the same symbol $\langle$, $\rangle$, is defined on the vector space $\left(T^{r} M\right)_{S}$ by the three equations

$$
\begin{aligned}
& <T_{1}^{v}, T_{2}^{v}>=<T_{1}, T_{2}>\circ \pi, \\
& <T^{v}, X^{n}>=0, \\
& <X_{1}^{h}, X_{2}^{n}>=<X_{1}, X_{2}>\circ \pi,
\end{aligned}
$$

where $X^{h}$ is the horizontal lift of $X$ with respect to $\nabla$. These equations are easily seen to determine $\bar{g}$ on $T^{r} M$ with respect to which the horizontal distribution $H$, induced by $\nabla$, is orthogonal to the fibres of $T^{r} M$ [3].

We now make use of $E$. For $X \in \mathscr{T}^{1}(M)$, define the vertical lift $X_{E}^{v}$ of $X$ with respect to $E$ by

$$
X_{E}^{v}=(E \otimes X)^{v}
$$

The map $X \rightarrow X_{E}^{v}$ is then a monomorphism of $\mathscr{T}^{1}(M) \rightarrow \mathscr{T}^{1}\left(T^{r} M\right)$. Hence an $n$-dimensional $C^{\infty}$ vertical distribution $V^{E}$ is defined on $T^{r} M$. Let $V^{\perp}$ be the distribution on $T^{r} M$ which is orthogonal to $H$ and $V^{E}$. Then $H, V^{E}$ and $V \perp$ are the required distributions and the proof is complete.

We now give an alternative characterization of $V \perp$.

Lemma 5. Let $p \in M, S \in \pi^{-1}(p)$, and $\mathscr{T}_{E}^{r}(p)$ be the subspace of $\mathscr{T}^{r}(p)$ defined by

$$
\mathscr{T}_{E}^{r}(p)=\left\{T:\left\langle T, E \otimes X>=0 \text { for all } X \in \mathscr{T}^{1}(p)\right\} .\right.
$$

Then $V_{S}^{\frac{1}{S}}=\left(\mathscr{T}_{E}^{r}(p)\right)_{S}^{v}$.

Let $E^{\perp}(p)$ be the subspace of $\mathscr{T}^{r-1}(p)$ defined by

$$
E^{\perp}(p)=\{T:\langle T, E\rangle=0\} .
$$

Then $\mathscr{T}_{E}^{r}(p)=E^{\perp}(p) \otimes \mathscr{T}^{\top}(p)$. 
Proof. The first part of the lemma follows from the fact that the vertical lift preserves scalar products. To prove the second part it is sufficient to note that $E^{\perp}(p) \otimes \mathscr{T}^{1}(p) \subset \mathscr{T}_{E}^{r}(p)$, and

$$
\operatorname{dim}\left(E^{\perp}(p) \otimes \mathscr{T}^{1}(p)\right)=n\left(n^{r-1}-1\right)=n^{r}-n=\operatorname{dim} \mathscr{T}_{E}^{r}(p) .
$$

Theorem. If $M$ admits an almost complex structure and a nowhere zero tensor field $E \in \mathscr{T}^{r-1}(M)$, then $T^{r} M$ admits an almost complex structure.

Proof. Let $F$ be an almost complex structure on $M$. We define a $C^{\infty}$ tensor field $J$ of type $(1,1)$ on $T^{r} M$ by its action on the distributions $H, V^{E}$ and $V^{\perp}$. Thus for $X \in \mathscr{T}^{1}(M)$ and $T \in \mathscr{T}^{r}(M)$ define $J$ by

$$
J\left(X^{h}\right)=X_{E}^{v}, J\left(X_{E}^{v}\right)=-X^{h}, J\left(T^{v}\right)=\bar{T}^{v},
$$

where $\bar{T}$ is obtained by contracting $T \otimes F$, and has components $T^{i_{1} i_{2} \cdots i_{r-1}{ }^{l}} F_{l}^{i_{r}}$, where $T^{i_{1} i_{2} \cdots i_{r}}$ and $F_{j}^{i}$ are local components of $T$ and $F$ respectively. The restrictions of $J$ to $H+V^{E}$ and $V^{\perp}$ are endomorphisms, and hence $J$ is a tensor field on $T^{r} M$. It is easily seen that $J$ is $C^{\infty}$ and $J^{2}=-I, I$ being the unit tensor. Hence $J$ is an almost complex structure on $T^{r} M$.

Corollary 12. Suppose a Riemannian manifold $M$ admits an almost complex structure. Then $T^{r} M$ admits an almost complex structure if (i) $r$ is ocid or (ii) $r$ is even and $M$ admits a nowhere zero vector field.

Proof. (i) For $r=2 s+1$ choose $E=\left(\otimes g^{-1}\right)^{s}$, where $g^{-1}$ is the inverse of a metric tensor field $g$ on $M$, and $\left(\otimes g^{-1}\right)^{s}$ is the tensor product of $g^{-1}$ with itself $s$ times.

(ii) For $r=2 s, s>1$, choose $E=\left(\otimes g^{-1}\right)^{s-1} \otimes X$, where $M$ is assumed to admit a nowhere zero vector field $X$. For $r=2$ choose $E=X$.

\section{Integrability of the almost complex structure $J$}

We now establish necessary and sufficient conditions for the integrability of $J$.

Let $e$ be the covariant tensor field of order $r-1$ associated to $E$ by $g$; thus, with respect to local coordinates, $e$ has components $e_{i_{1} i_{2} \ldots i_{r-1}}$ given by

$$
e_{i_{1} i_{2} \cdots i_{r-1}}=g_{i_{1} j_{1}} g_{i_{2} j_{2} \ldots} g_{i_{r-1} j_{r-1}} E^{j_{1} j_{2} \cdots j_{r-1}} \text {. }
$$

Proposition 3. Suppose $M$ admits an almost complex structure $F$ and $a$ nowhere zero tensor field $E \in \mathscr{T}^{r-1}(M)$. Then the induced almost complex structure $J$ is integrable if and only if, for $X, Y \in \mathscr{T}^{\mathrm{J}}(M)$,

$$
R(X, Y)=0, \quad \nabla_{X} E=0, \quad \nabla_{X} F=0, \quad \nabla_{X} \frac{e}{<E, E>}=0
$$


Proof. Let $N$ be the Nijenhuis 2-form on $T^{r} M$ with values in $\mathscr{T}^{1}\left(T^{r} M\right)$, defined by

$$
N\left(W_{1}, W_{2}\right)=\left[W_{1}, W_{2}\right]+J\left[J W_{1}, W_{2}\right]+J\left[W_{1}, J W_{2}\right]-\left[J W_{1}, J W_{2}\right]
$$

for $W_{1}, W_{2} \in \mathscr{T}^{1}\left(T^{r} M\right)$. Then $J$ is integrable if and only if $N=0$.

Suppose $N=0$. Then for $X, Y \in \mathscr{T}^{1}(M), N\left(X_{E}^{v}, Y_{E}^{v}\right)=0$. Hence, putting $W_{1}=X_{E}^{v}, W_{2}=Y_{E}^{v}$ we have, from (9), (12), (13), and the definition of $J$,

$$
\begin{aligned}
\overline{R(X, Y)} & =J\left(\nabla_{Y}(E \otimes X)\right)^{v}-J\left(\nabla_{X}(E \otimes Y)\right)^{v}-[X, Y]^{h} \\
& =J\left(\left(\nabla_{Y} E\right) \otimes X\right)^{v}-J\left(\left(\nabla_{X} E\right) \otimes Y\right)^{v}-\left(\nabla_{Y} X\right)^{h} \\
& +\left(\nabla_{X} Y\right)^{h}-[X, Y]^{h} \\
& =J\left(\left(\nabla_{Y} E\right) \otimes X-\left(\nabla_{X} E\right) \otimes Y\right)^{v}
\end{aligned}
$$

since $\nabla$ has zero torsion. Now since $E \otimes \mathscr{T}^{1}(M)$ is a subspace of $\mathscr{T}^{r}(M)$ there is a unique $T \in \mathscr{T}^{r}(M)$ orthogonal to this subspace and a unique $Z \in \mathscr{T}^{1}(M)$ such that

$$
\left(\nabla_{Y} E\right) \otimes X-\left(\nabla_{X} E\right) \otimes Y=T+E \otimes Z .
$$

Then from (19) and (20)

$$
\overline{R(X, Y)}=\bar{T}^{v}-Z^{n} .
$$

Since $\overline{R(X, Y)}$ is vertical, $Z^{h}=0$ and hence $Z=0$. It follows from Corollary 11 that

$$
\begin{gathered}
R(X, Y)=0, \\
T=0 .
\end{gathered}
$$

We thus have for all $X, Y \in \mathscr{T}^{1}(M)$,

$$
\left(\nabla_{X} E\right) \otimes Y=\left(\nabla_{Y} E\right) \otimes X .
$$

Since $M$ is assumed to admit an almost complex structure, $\operatorname{dim} M \geq 2$. Hence by choosing $X, Y$ to be linearly independent it follows that

$$
\nabla_{X} E=0 \text {. }
$$

We next consider the case $N\left(X_{E}^{v}, T^{v}\right)=0$, where $X_{E}^{v} \in V^{E}$ and $T^{v} \in V^{\perp}$. Then from (9), (12) and the definition of $J$ we have

$$
J\left(\nabla_{X} T\right)^{v}=\left(\nabla_{X} \bar{T}\right)^{v} .
$$

It follows that $\left(\nabla_{X} T\right)^{v} \in V^{\perp}$. Choose $T=S \otimes Y$ where $S \in \mathscr{T}^{r-1}(M), Y \in \mathscr{T}^{1}(M)$ 
and $\langle S, E\rangle=0$ (since $M$ is paracompact such an $S$ exists and can be chosen to be non-zero in a neighbourhood of a point). Then by Lemma 5 , $T^{v} \in V \perp$ and (24) imply that

$$
\left(\nabla_{X} S\right) \otimes F Y+S \otimes F \nabla_{X} Y=\left(\nabla_{X} S\right) \otimes F Y+S \otimes \nabla_{X}(F Y) .
$$

Hence

$$
S \otimes\left(\nabla_{X} F\right) Y=0
$$

and it follows immediately that

$$
\nabla_{X} F=0 \text {. }
$$

Finally, from Lemma 5 the condition $\left(\nabla_{X} T\right)^{v} \in V^{\perp}$ implies that

$$
0=e\left(\nabla_{X} S\right)=-\left(\nabla_{X} e\right) S .
$$

But $S$ is any tensor field which satisfies $\langle S, E\rangle=0$. Hence we deduce that

$$
\nabla_{X} e=\alpha(X) e,
$$

where $\alpha \in \mathscr{T}_{1}(M)$. Then $\alpha$ is determined by

$$
\alpha(X)=\frac{\left(\nabla_{X} e\right)(E)}{e(E)}=\frac{X(e(E))}{e(E)}=\frac{X<E, E>}{\langle E, E>} .
$$

Thus

$$
\alpha=d \log e(E)=d \log \langle E, E\rangle .
$$

(If $V$ is the Riemannian connection associated with $g$ then (23) implies (27) and $\alpha=0$.) Hence, from (27) and (28), the tensor field $\frac{e}{\langle E, E\rangle}$ has zero covariant derivative. This proves the necessity of the conditions in Proposition 3 .

To prove the sufficiency we note that

$$
\begin{aligned}
& N\left(X_{E}^{v}, Y_{E}^{v}\right)=N\left(Y^{h}, X^{n}\right)=J N\left(Y_{E}^{v}, X^{h}\right), \\
& N\left(X_{E}^{v}, T^{v}\right)=J N\left(T^{v}, X^{n}\right), \quad N\left(T_{1}^{v}, T_{2}^{v}\right)=0 .
\end{aligned}
$$

Thus $N=0$ if $N\left(X_{E}^{v}, Y_{E}^{v}\right)=N\left(X_{E}^{v}, T^{v}\right)=0$. Suppose $\nabla_{X} E=0$ and $R(X, Y)=0$ for all $X, Y \in \mathscr{T}^{1}(M)$. Then

$$
\begin{aligned}
N\left(X_{E}^{v}, Y_{E}^{v}\right) & =-J\left[X^{h}, Y_{E}^{v}\right]-J\left[X_{E}^{v}, Y^{h}\right]-\left[X^{h}, Y^{h}\right] \\
& =\left(V_{X} Y\right)^{h}-\left(V_{Y} X\right)^{h}-[X, Y]^{h}=0 .
\end{aligned}
$$


Suppose $\nabla_{X} \frac{e}{\langle E, E\rangle}=0$. Then (27) follows and hence if $T^{v} \in V^{\perp}$ then $\left(V_{X} T\right)^{v} \in V^{\perp}$. If we next assume $\nabla_{X} F=0$ then we have

$$
N\left(X_{E}^{v}, T^{v}\right)=\left(\nabla_{X} \bar{T}\right)^{v}-J\left(\nabla_{X} T\right)^{v}=0,
$$

which proves the sufficiency.

\section{Kählerian structure on $T^{\tau} M$}

We now determine necessary and sufficient conditions for the metric $\bar{g}$ on $T^{r} M$, defined in $\S 5$, to be Kählerian with respect to $J$.

Proposition 4. $\bar{g}$ is Hermitian with respect to $J$ if and only if $\langle E, E\rangle=1$ and $g$ is Hermitian with respect to $F$.

Proof. Suppose $\bar{g}$ is Hermitian with respect to $J$. Then for $X, Y \in \mathscr{T}^{\mathrm{J}}(M)$,

$$
\begin{aligned}
\langle X, Y>\circ \pi & =\left\langle X^{h}, Y^{h}\right\rangle=\left\langle J X_{E}^{v}, J Y_{E}^{v}\right\rangle=\left\langle X_{E}^{v}, Y_{E}^{v}\right\rangle \\
& =\langle E \otimes X, E \otimes Y>\circ \pi=\langle E, E\rangle\langle X, Y>\circ \pi .
\end{aligned}
$$

Hence $\langle E, E\rangle=1$. Now let $p \in M$ and let $S \in \mathscr{T}^{r-1}(p)$ be non-zero such that $\langle S, E\rangle=0$. Then by Lemma 5 and the definition of $J$ we have, for $X, Y \in \mathscr{T}^{1}(p)$,

$$
\begin{aligned}
& <S, S>\langle X, Y>\circ \pi=\langle S \otimes X, S \otimes Y>\circ \pi \\
& =\left\langle(S \otimes X)^{v},(S \otimes Y)^{v}>=\left\langle J(S \otimes X)^{v}, J(S \otimes Y)^{v}\right\rangle\right. \\
& =\langle S \otimes F X, S \otimes F Y>\circ \pi=\langle S, S>\langle F X, F Y>\circ \pi .
\end{aligned}
$$

Thus at $p,\langle X, Y\rangle=\langle F X, F Y\rangle$. Since $p$ is arbitrary, $g$ is Hermitian with respect to $F$. The sufficiency of the above conditions is easily proved by the same method.

Proposition 5. Suppose $\bar{g}$ is Hermitian with respect to J. Then $\bar{g}$ is Kählerian with respect to $J$ if and only if $\nabla$ is the Riemannian connection associated with $g, R=0, \nabla E=0$ and $\nabla F=0$.

Proof. Let $\alpha$ be the field of 2-forms on $T^{r} M$ defined for all $W_{1}, W_{2} \in \mathscr{T}^{1}\left(T^{r} M\right)$ by $\alpha\left(W_{1}, W_{2}\right)=\left\langle W_{1}, J W_{2}\right\rangle$. Then $\bar{g}$ is Kählerian with respect to $J$ if and only if $\alpha$ is closed and $J$ is integrable [6, Chapter VII]. As usual it is sufficient to consider the action of $\alpha$ and $d \alpha$ on the three distributions $H, V^{E}$ and $V^{\perp}$ on $T^{r} M$. Then for $X, Y \in \mathscr{T}^{1}(M)$ and $T_{1}^{v}, T_{2}^{v} \in V^{\perp}$ we have

$$
\begin{aligned}
& \alpha\left(X_{E}^{v}, Y_{E}^{v}\right)=\alpha\left(X^{h}, Y^{h}\right)=\alpha\left(T_{1}^{v}, X_{E}^{v}\right)=\alpha\left(T_{1}^{v}, X^{h}\right)=0, \\
& \alpha\left(X_{E}^{v}, Y^{h}\right)=\langle E \otimes X, E \otimes Y>\circ \pi=<X, Y>\circ \pi, \\
& \alpha\left(T_{1}^{v}, T_{2}^{v}\right)=<T_{1}, \grave{T}_{2}>\circ \pi .
\end{aligned}
$$


Suppose $\bar{g}$ is Kählerian with respect to $J$. Then by Propositions 3 and 4 , $R=0, \nabla_{X} E=0$, and $\nabla_{X} e=0$, for all $X \in \mathscr{T}^{1}(M)$. Let $p \in M, X \in \mathscr{T}^{1}(p)$, and choose $T \in \mathscr{T}^{r-1}(M)$ such that $\langle T, E\rangle=0$ and $\langle T, T\rangle=1$ on some neighbourhood $U$ of $p$. Since $R=0$ parallel vector fields $Y$ and $Z$ exist on $U$ with arbitrary initial values at $p$. Then using (9), (12) and Lemma 5 we have, on $\pi^{-1}(p)$,

$$
\begin{aligned}
& 0=d \alpha\left((T \otimes Y)^{v},(T \otimes X)^{v}, X^{h}\right) \\
& =X\left\langle T \otimes Y, T \otimes F X>+\left\langle T \otimes F Y, \nabla_{X}(T \otimes Z)\right\rangle\right. \\
& -\left\langle\nabla_{X}(T \otimes Y), T \otimes F Z>\right. \\
& =X\langle Y, F Z\rangle+2\left\langle T, \nabla_{X} T\right\rangle\langle F Y, Z\rangle \\
& +\left\langle F Y, \nabla_{X} Z\right\rangle-\left\langle\nabla_{X} Y, F Z\right\rangle \\
& =\left(\nabla_{X} g\right)(Y, F Z)-2\left\langle T, \nabla_{X} T\right\rangle\langle Y, F Z\rangle \text {. }
\end{aligned}
$$

Since $F$ is non-singular it follows that

$$
\nabla_{X} g=\alpha(X) g
$$

for some $\alpha \in \mathscr{T}_{1}(p)$. Then since $\nabla_{X} E=0$ and $\nabla_{X} e=0$ it follows easily that for all $X \in \mathscr{T}^{1}(p)$,

$$
0=\nabla_{X} e=(r-1) \alpha(X) e .
$$

The tensor $e$ is non-zero and so $\alpha=0$. Thus $\nabla g=0$ at $p$ and hence on $M$ since $p$ is arbitrary. It follows that $\nabla$, having no torsion, is the Riemannian connection associated with $g$.

We now prove the sufficiency of the above conditions by showing that the 2-form $\alpha$ is exact. Let $X \in \mathscr{T}^{1}(M)$, and $T^{v} \in V^{\perp}$. Define a 1 -form $\beta$ on $T^{r} M$ as follows: at each point $S \in T^{r} M$,

$$
\beta\left(X^{h}\right)=\langle S, E \otimes X\rangle, \quad \beta\left(X_{E}^{v}\right)=0, \quad \beta\left(T^{v}\right)=\frac{1}{2}\langle S, \bar{T}\rangle .
$$

Then using (29) it follows after some calculation that $\alpha=d \beta$. Hence $d \alpha=0$, and this together with Proposition 3 proves the sufficiency.

\section{Integrability of $H+V^{E}$ and $H+V^{\perp}$}

Proposition 6. $H+V^{E}$ is integrable if and only if $R=0$ and for $X \in \mathscr{T}^{1}(M)$, $\nabla_{X} E=\alpha(X) E$, where $\alpha(X)=\frac{\left\langle E, \nabla_{X} E\right\rangle}{\langle E, E\rangle}$.

Proof. It follows from (12) and (13) that $H+V^{E}$ is an integrable distribution if and only if for $X_{1}, X_{2} \in \mathscr{T}^{1}(M)$,

$$
\left(\nabla_{X_{1}}\left(E \otimes X_{2}\right)\right)^{v} \in V^{E},
$$




$$
\overline{R\left(X_{1}, X_{2}\right)} \in V^{E} .
$$

Let $Y_{1}$ and $Y_{2}$ be orthogonal vectors at $p \leqslant M$, and let $\langle T, E\rangle=0$ at $p$. Then from (16), (32) and Corollary 6,

$$
\begin{aligned}
0 & =\left\langle R\left(X_{1}, X_{2}\right)\left(T \otimes Y_{1}\right), T \otimes Y_{2}>\right. \\
& =<T, T><R\left(X_{1}, X_{2}\right) Y_{1}, Y_{2}>.
\end{aligned}
$$

Hence $R\left(X_{1}, X_{2}\right) Y_{1}=c Y_{1}$ where $c$ is some real number which depends on $X_{1}$ and $X_{2}$. Since $Y_{1}$ is arbitrary it follows that $R\left(X_{1}, X_{2}\right)=c I$ at $p$. Then at any point $S \in \pi^{-1}(p)$ we have $\overline{R\left(X_{1}, X_{2}\right)}=-c r S^{v}$, and by choosing $S^{v} \in V^{\perp}$ it follows that $\overline{R\left(X_{1}, X_{2}\right)}=0$ at $S$; hence $c=0$. Since $p, X_{1}$ and $X_{2}$ are arbitrary we have $R=0$ on $M$.

Using (30) and Lemma 5 we obtain $\nabla_{X} E=\alpha(X) E$ and $\alpha$ is then uniquely determined by this equation.

The proof of the sufficiency is immediate.

Proposition 7. $H+V^{\perp}$ is integrable if and only if $R=0$ and for $X \in \mathscr{T}^{1}(M)$, $\nabla_{X} e=\alpha(X) e$, where $\alpha=\frac{\left\langle e, \nabla_{X} e\right\rangle}{\langle e, e\rangle}$.

Proof. The proof is similar to that of Proposition 6 and we shall use the same notation. It follows from (12), (13) and Lemma 5 that $H+V \perp$ is an integrable distribution if and only if for $S^{v} \in V^{\perp}$,

$$
\begin{gathered}
\left(\nabla_{X_{1}}\left(S \otimes X_{2}\right)\right)^{v} \in V^{\perp}, \\
\overline{R\left(X_{1}, X_{2}\right)} \in V^{\perp} .
\end{gathered}
$$

then from (16), (34) and Corollary 6 ,

$$
\begin{aligned}
0 & =\left\langle R\left(X_{1}, X_{2}\right)\left(E \otimes Y_{1}\right), E \otimes Y_{2}>\right. \\
& =<E, E><R\left(X_{1}, X_{2}\right) Y_{1}, Y_{2}>.
\end{aligned}
$$

Hence, as before, $R=0$.

From (33) we obtain

$$
0=\left\langle\nabla_{X_{1}} S, E\right\rangle\left\langle X_{2}, Y\right\rangle
$$

for $Y \in \mathscr{T}^{1}(p)$. Hence

$$
0=\left\langle\nabla_{X_{1}} S, E\right\rangle=e\left(\nabla_{X_{1}} S\right)=-\left(\nabla_{X_{1}} e\right) S .
$$

It follows that $\nabla_{X_{1}} e=\alpha\left(X_{1}\right) e$ at $p$. Since $p$ and $X_{1}$ are arbitrary we obtain $\nabla_{X_{1}} e=\alpha\left(X_{1}\right) e$ on $M$, and $\alpha$ is then uniquely determined.

The proof of the sufficiency is immediate. 


\section{References}

[1] P. Dombrowski, On the goemetry of tangent bundles, J. Reine Angew. Math. 210 (1962) 73-88.

[2] S. Helgason, Differential geometry and symmetric spaces, Academic Press, New York, 1962.

[3] S. Sasaki, On the differential geometry of tangent bund!es of Riemannian manifolds, Tôhoku Math. J. 10 (1958) 338-354.

[4] A. J. Ledger \& K. Yano, The tangent bundle of a locally symmetric space, J. London Math. Soc. 40 (1965) 487-492.

[5] S. Tachibana \& M. Okumura, On the almost complex structure of tangent bundles of Riemannian spaces, Tôhoku Math. J. 14 (1962) 158-161.

[6] K. Yano, Differential geometry on complex and almost complex spaces, Pergamon, New York, 1965.

[7] K. Yano \& S. Ishihara, Almost complex structures induced in tangent bundles, Kōdai Math. Sem. Rep. 19 (1967) 1-27.

[8] K. Yano \& S. Kobayashi, Prolongations of tensor fields and connections to tangent bundles I, J. Math. Soc. Japan 18 (1966) 194-210.

[9] K. Yano \& A. J. Ledger, Linear connections on tangent bundles, J. London Math. Soc. 39 (1964) 495-500.

[10] K. Yano \& E. M. Patterson, Vertical and complete lifts from a manifold to its cotangent bundle, J. Math. Soc. Japan 19 (1967) 91-113.

UNIVERSITY OF LIVERPOOL

TOKYO INSTITUTE OF TECHNOLOGY 